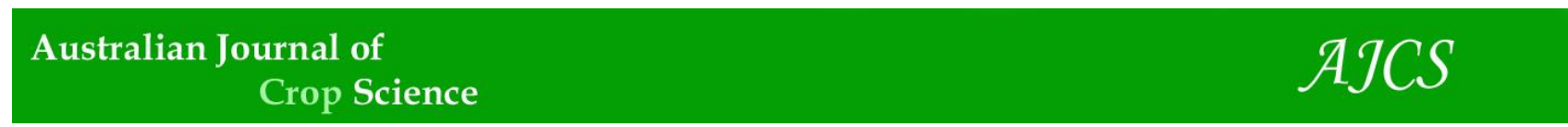

AJCS 11(07):853-860 (2017)

ISSN:1835-2707

doi: 10.21475/ajcs.17.11.07.pne505

\title{
Enhanced tolerance to salinity stress and ABA is regulated by Oryza sativa STRESS ASSOCIATED PROTEIN 8 (OSSAP8)
}

\author{
Nur Farhana Roslan ${ }^{1}$, Nor Syafiqah Abd Rashid ${ }^{1}$, Intan Elya Suka ${ }^{1}$, Nadiatul Ain Nabila Ahmad \\ Taufik $^{1}$, Nur Syahirah Abdullah ${ }^{1}$, Mohd Bajuri Asruri ${ }^{1}$, Bernadette Toni ${ }^{2}$, Noor Liyana Sukiran ${ }^{1}$, \\ Zamri Zainal ${ }^{1,2}$, Nurulhikma Md Isa ${ }^{1}$ *
}

\author{
${ }^{1}$ School of Biosciences and Biotechnology, Faculty of Sciences and Technology, Universiti Kebangsaan Malaysia, \\ 43600 UKM Bangi, Selangor, Malaysia \\ ${ }^{2}$ Institute of Systems Biology (INBIOSIS), Universiti Kebangsaan Malaysia, 43600 UKM Bangi, Selangor, \\ Malaysia
}

*Corresponding author: hikma@ukm.edu.my

\begin{abstract}
Increased tolerance to multiple abiotic stresses in plants is a key target for food security. OsSAP8 is a member of the stress-associated protein (SAP) gene family in rice and is characterized by the presence of two zinc finger domains, A20 and AN1. In this research, we aim to understand the regulatory mechanism of $O S S A P 8$ in response to multiple stresses for rice survival. We used the Arabidopsis transformation system to understand the gene's function. Two Arabidopsis lines were used; OsSAP8 overexpression line and Arabidopsis atsap2 knockout loss-of-function mutation in comparison to wild type Columbia-0 (Col-0). The protein sequence of Arabidopsis atsap2 has been shown to be highly identical to the OSSAP8. An atsap2 knockout showed a sensitive phenotype to high concentration of $\mathrm{NaCl}$ and PEG with reduced germination percentage. OsSAP8 overexpression showed a tolerance phenotype to 150 $\mathrm{mM} \mathrm{NaCl}$ and 20\% PEG compared to atsap2. OsSAP8 also showed an insensitive germination phenotype to high concentration of ABA compared to Col-0 and atsap2. The insensitive phenotype of OsSAP8 on the high concentration of ABA (12 $\mu \mathrm{M})$ and the sensitive phenotype of its orthologue atsap2 in Arabidopsis suggests that OsSAP8 may have a role in part through ABA signaling as a putative transcriptional regulator for $\mathrm{ABA}$ during stress response.
\end{abstract}

Keywords: abiotic stress, Arabidopsis, atsap2 gene, Oryza sativa, OsSAP8 gene, seed germination. Abbreviations: ABA_Abscisic acid; $\mathrm{NaCl}$ _Sodium chloride; PEG_Polyethylene glycol.

\section{Introduction}

Agricultural productivity is a major challenge due to the increasing world population and the associated high demand for food production (Godfray et al., 2010). Abiotic stresses have been identified as a major threat to the farmer as a result of reduced crop yields. These stresses include drought, salinity, flood, heat and cold, and are mainly related to global climate change and adversely affect crop productivity. High salinity in soil negatively impacts crop growth, development, yield quality and quantity due to an excess of $\mathrm{Na}^{+}$and $\mathrm{Cl}^{-}$ ions (Deinlein et al., 2014; Munns and Tester 2008; Munns, 2002). This occurs by impairing metabolic processes, osmotic content, nutritional balance in plants and decreasing photosynthetic efficiency (Tester and Davenport, 2003). Plants have specific mechanisms to retain resources for growth and development in response to environmental stress (Atkinson et al., 2015).

Genetic engineering has been used for agricultural improvement by producing crops that are resistance to stress. Transgenic approaches have been used widely in order to understand the mechanism of plant responses to abiotic stress (Vij and Tyagi, 2007; Cattivelli et al., 2008). This study has employed the genetic engineering approach to understand the molecular mechanism underlying abiotic stresses. The Stress Associated Protein (SAP) family, which is known to have an important role in multiple abiotic stress response in plants, comprises 18 and 14 gene members that have been identified in Oryza sativa and Arabidopsis thaliana genomes, respectively. One of the members from the rice SAP gene family, OSSAP 8 has been identified to be differentially upregulated in our salt-stress subtractive hybridization ( $\mathrm{SSH}$ ) library (Hedayati et al., 2015). OsSAP8 is well known as having multiple stress inducible genes. These stresses include heat, cold, salt, desiccation, submergence, wounding and heavy metals (Kanneganti and Gupta, 2008). OsSAP8 is characterized by the presence of two types of zinc finger domains, A20 and AN1. The A20 domain is embedded in the $\mathrm{N}$-terminal of the coding region whilst the AN1 domain is embedded near the C-terminal of the coding sequence (Martin et al., 2012).

Abscisic acid (ABA) is a major plant phytohormone involved in stress response. Previous studies have dissected the core signaling pathway for ABA including the receptors and all other components (Nambara and Marion-Poll, 2005; Nambara et al., 2010). Several different classes of ABA receptors have been reported. PYRABACTIN RESISTANCE 1 (PYR1) was identified as an ABA receptor through a chemical genetic approach (Park et al., 2009). The Arabidopsis genome encodes 13 genes that have similarity to 
PYR1, which were named PYR1 LIKE-13 (PYL1-13). These genes are alternatively named REGULATORY COMPONENTS OF ABA RECEPTOR (RCARs) (Ma et al., 2009). All PYR1/PYL1-PYL13/RCARs belong to the same gene family in Arabidopsis (Umezawa et al., 2010; Park et al., 2009; Ma et al., 2009). Protein phosphatase 2C (PP2C) is a member of the protein serine/threonine phosphatases. PP2C has been shown to have important roles in ABA signaling, acting as a negative regulator. ABSCISIC ACID INSENSITIVE1, 2 (ABI1, ABI2), which were identified based on their insensitivity to ABA have been shown to encode paralogous PP2Cs (Leung et al., 1997; Meyer et al., 1994; Leung et al., 1994). Positive regulators of ABA signaling which act downstream of the PP2Cs have been identified as SNF-1 related protein kinase 2s (SnRK2s) (Nambara et al., 2010 \& Umezawa et al., 2010). There are 10 SnRK protein kinases in Arabidopsis, divided into 3 subclasses (Kobayashi et al., 2004). Each subclass has a different response to a different abiotic stress. Subclass I SnRK2s for example are strongly activated by an osmotic stress but they are not activated by ABA, whilst Subclass II and III are activated by both osmotic stress and ABA and only Subclass III has a strong response to ABA (Umezawa et al., 2010). This finding has encouraged many researchers to explore more the relationship between $\mathrm{ABA}$ and other potential transcriptional regulators in response to environmental stresses.

In this study, we provide an understanding of OsSAP8 regulation under different stress conditions including $\mathrm{ABA}$. Overexpression of $O S S A P 8$ resulted in tolerance to high concentrations of $\mathrm{NaCl}$ compared to its knockout mutant atsap2; this result was observed through its high germination score, assessed on day 7. Interestingly, OsSAP8 showed an insensitive phenotype to high concentrations of $\mathrm{ABA}$ up to $12 \mu \mathrm{M}$ compared to the knockout mutant atsap2 and wild type Col-0. This suggests that OsSAP8 may play a role as a putative transcriptional regulator of $\mathrm{ABA}$ during plant response to abiotic stress. However, more studies need to be performed to determine the relationship between OsSAP8 and ABA signaling.

\section{Results}

\section{Analysis of OsSAP8 positive transformants and atsap2 mutants}

Seeds harvested from transformed plants were screened on $150 \mathrm{mg} / \mathrm{ml}$ BASTA. Surviving plants were positive transformants as they carry the pB2GW7-OsSAP8 construct. PCR on the genomic DNA showed fragments with a size of $\sim 519$ bp which indicates the presence of OSSAP8 in two out of four transformed lines (Fig. 1). Verification of atsap2 knock-out mutant line was confirmed through semiquantitative RT-PCR. We verified that this homozygous knock-out mutant has a null transcription by semiquantitative RT-PCR. The semi-quantitative RT-PCR result showed that there is no transcript expression of atsap2 identified in all the atsap2 knock-out mutant plants (Fig. 2), while only Col- 0 showed the expression of atsap 2 with a size of $288 \mathrm{bp}$.

\section{Germination of OsSAP8 overexpression seeds is more tolerant to $\mathrm{NaCl}$}

In order to understand the role of $O S S A P 8$ in regulating saltstress, germination analysis of $O S S A P 8$ at different $\mathrm{NaCl}$ concentrations was carried out. Seeds used in this experiment were after-ripened. A controlled experiment was performed on water agar media to observe seed viability for both seeds used in $\mathrm{NaCl}$ and PEG tests (Figs. 3 and 5). Germination response revealed that $O S S A P 8$ seeds were tolerant to $\mathrm{NaCl}$ up to $150 \mathrm{mM}$, with $98 \%$ of seeds being germinated compared to $83 \%$ for Col-0 (Fig. 3). In fact, OsSAP8 showed rapid germination on all media supplemented by $\mathrm{NaCl}$. This can be observed on day 1 , where seeds of OSSAP8 germinated almost $90 \%$ on media supplemented by up to 75 $\mathrm{mM} \mathrm{NaCl}$, whilst a fast germination rate can be observed on day 2 for media supplemented with $150 \mathrm{mM} \mathrm{NaCl}$ compared to atsap2 and Col-0 (Fig. 3). Instead of OSSAP8, we also carried out a germination test on atsap2 T-DNA knockout seeds. atsap2 is the orthologue gene of OsSAP8 in Arabidopsis (Fig. 4). Both OsSAP8 and atsap2 consist of the same zinc finger domain A20 and AN1. To investigate whether the presence of Stress Associated Protein is important during salt-stress, we carried out the same germination test on atsap2 knockout mutants. Results showed that atsap2 germination is impaired on media supplemented with $150 \mathrm{mM} \mathrm{NaCl}$, with $47 \%$ germination score compared to wild type Col-0, $83 \%$. This indicates that in the knockout atsap2, the expression of Stress Associated Protein 2 is completely abolished.

Other than salt-stress, we also carried out research on PEG osmotic stress. This was performed using various PEG-6000 concentrations $(10 \%, 20 \%, 40 \%, 50 \%)$ and water agar media as control experiment. Both OsSAP8 and atsap2 seeds were plated onto various concentrations of PEG, with Col-0 acting as control. Germination curves showed that OSSAP8 seeds were germinated more rapidly than atsap 2 and Col- 0 on $10 \%$ and 20\% PEG-6000, which can be observed on day two (Fig. 5). Overall, OsSAP 8 showed rapid germination but as the imbibition day prolonged to day seven, germination percentage for both $O S S A P 8$ and Col-0 did not significantly differ (Fig. 5). However, germination percentage for OSSAP8 was much higher compared to atsap2 in all media supplemented with PEG (Fig. 5). Both results from $\mathrm{NaCl}$ and PEG indicate that overexpression of OsSAP8 enhanced seeds' tolerance to salt but not osmotic stress, whilst the knockout mutant seed of atsap 2 showed a susceptible phenotype to both stresses.

\section{Germination of OsSAP8 overexpression seeds is $A B A$ insensitive}

To investigate whether ABA affected germination potential in OsSAP8 and atsap2, a germination assay was carried out at different $\mathrm{ABA}$ concentrations over seven days. atsap2 and Col-0 germination were relatively sensitive at $12 \mu \mathrm{M} \mathrm{ABA}$ with $16 \%$ and $30 \%$ seed germination, respectively, while OsSAP8 showed an insensitive germination phenotype on 12 $\mu \mathrm{M}$ ABA with $84 \%$ seed germination (Fig. 6). Besides, OsSAP8 showed a faster germination rate compared to atsap2 and Col-0 on all media supplemented with different ABA concentrations $(2 \mu \mathrm{M}, 5 \mu \mathrm{M}, 7 \mu \mathrm{M}, 10 \mu \mathrm{M}$ and $12 \mu \mathrm{M}$ ) (Fig. 6). This indicates that the overexpression of OsSAP8 gene in Arabidopsis made the seed insensitive to high concentrations of $\mathrm{ABA}$, while the knockout version of atsap2 showed a sensitive germination phenotype to ABA (Fig. 6).

\section{Rice abiotic stress co-expression network for OSSAP8}

Further understanding in terms of how $O S S A P 8$ relates to other abiotic stress genes is important. This allows us to interpret the regulatory mechanism of OSSAP8 in relation to other genes during stress situations. A co-expressed network 
Table 1. List of Co-expressed genes, Table representing six genes co-expressed with OsSAP8. Detail of the Rice ID is shown in the table with the gene annotation for each.

\begin{tabular}{lc}
\hline Rice ID & Annotation \\
\hline LOC_Os05g48800 & drought induced 19 protein, putative, expressed \\
LOC_Os04g57220 & ubiquitin-conjugating enzyme, putative, expressed \\
LOC_Os11g43900 & translationally-controlled tumor protein, putative, expressed \\
LOC_Os07g34589 & translation initiation factor SUI1, putative, expressed \\
LOC_Os12g43600 & RNA recognition motif containing protein, expressed \\
LOC_Os03g46770 & RNA recognition motif containing protein, expressed \\
\hline
\end{tabular}

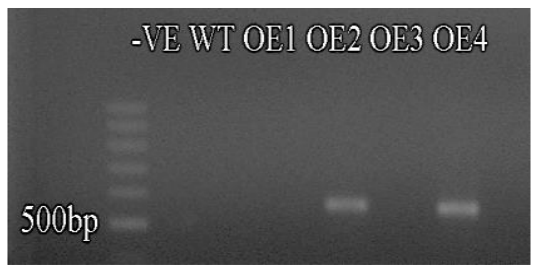

Fig 1. 35S::OsSAP8 positive transformants were screened via PCR. Agarose gel electrophoresis of polymerase chain reaction (PCR) shows the presence of full length sequence of OSSAP8 ( $519 \mathrm{bp}$ ) in overexpression Arabidopsis thaliana transgenic plants (lane OE2 and OE4). Ladder indicates 100bp ladder, -VE indicates negative control, WT indicates genomic DNA of wild type (Col- 0$)$ and OE indicates overexpression lines.

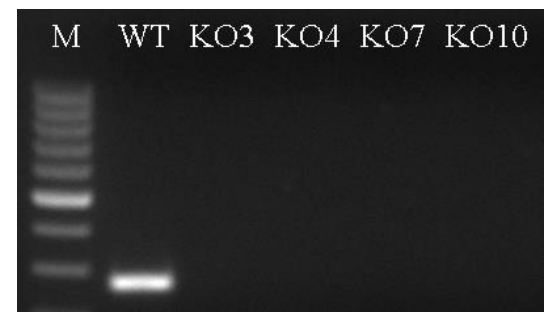

Fig 2. Verification of atsap2 expression in T-DNA mutant lines. Expression of atsap2 was confirmed by semi-quantitative reverse transcriptase PCR (RT-PCR). Reverse-transcription PCR result shows that atsap2 only expressed in Col-0 indicated by one single band with a size of $288 \mathrm{bp}$, but not in all T-DNA knockout mutant lines. M indicates 100bp marker, WT indicates wild-type Col-0 and $\mathrm{KO}$ indicates T-DNA knockout mutants (KO3, KO4, KO7, KO10).
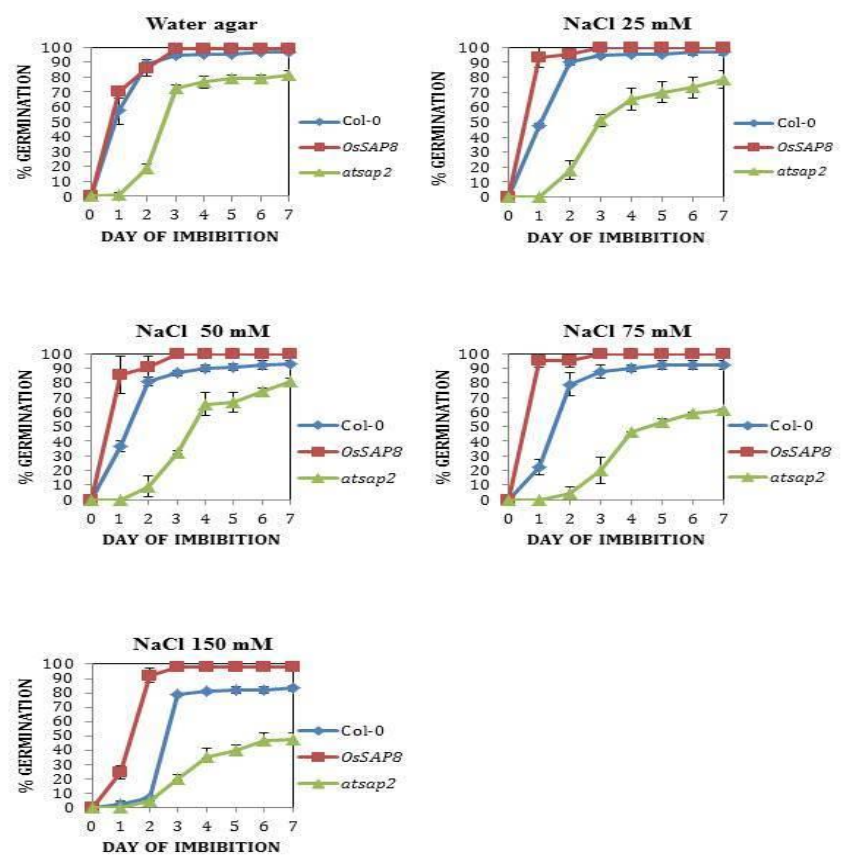

Fig 3. Germination analysis was carried out for Col-0, OsSAP8 and atsap2 in response to different concentrations of $\mathrm{NaCl}$. The graph shows the effect of different $\mathrm{NaCl}$ concentrations $(25 \mathrm{mM}, 50 \mathrm{mM}, 75 \mathrm{mM}$ and $100 \mathrm{mM})$ on the germination of Col-0, OsSAP8 and atsap 2 seeds over seven days. Water agar was used as a control experiment to observed seed viability. atsap 2 showed a sensitive germination phenotype to high concentrations of $\mathrm{NaCl}$ up to $150 \mathrm{mM}$ compared to Col-0 and OsSAP8. OsSAP8 showed a tolerant phenotype to high concentrations of $\mathrm{NaCl}$ and rapid germination rate on day 1 in media supplemented with $25 \mathrm{mM}, 50 \mathrm{mM}$ and 75 $\mathrm{mM}$ compared to atsap2 and Col-0. 


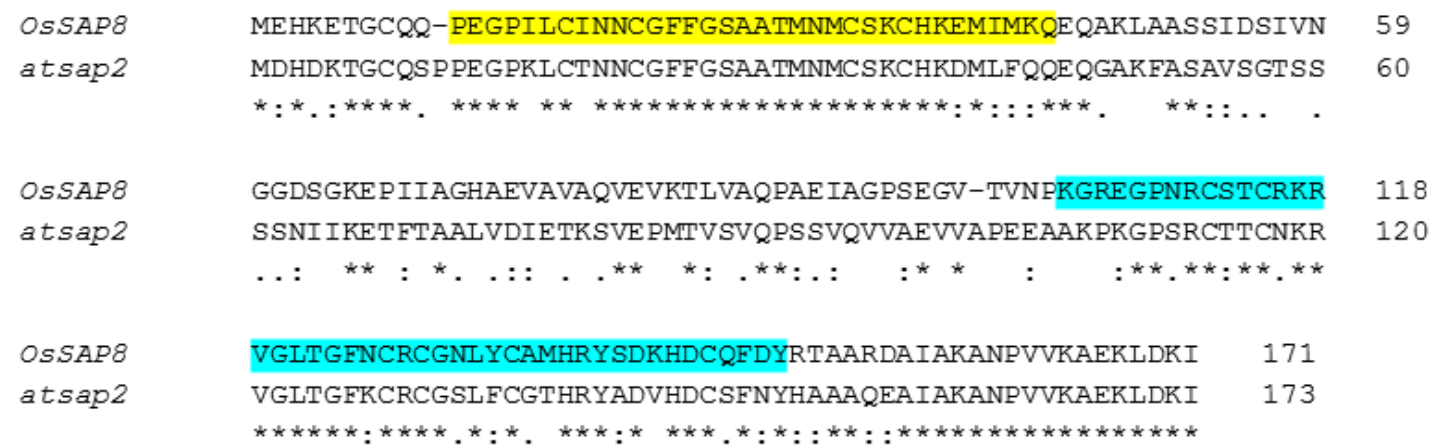

Fig 4. Protein sequence alignment for both $O S S A P 8$ and atsap 2 was carried out using Clustal Omega software. Highlighted in yellow is the A20 domain, while highlighted in blue is the AN1 domain. These two domains are highly conserved in both $O s S A P 8$ and atsap 2 protein sequences. These two zinc-finger domains were identified to be involved in stress response.

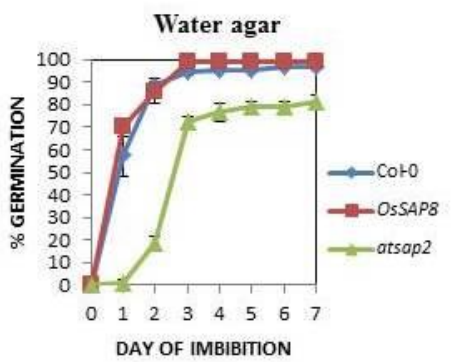

$20 \%$ PEG

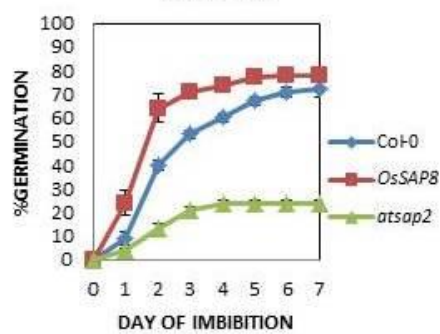

$50 \%$ PEG

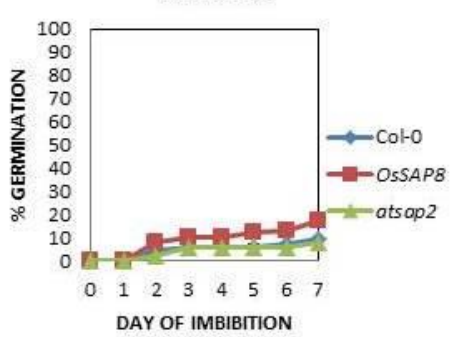

$10 \%$ PEG

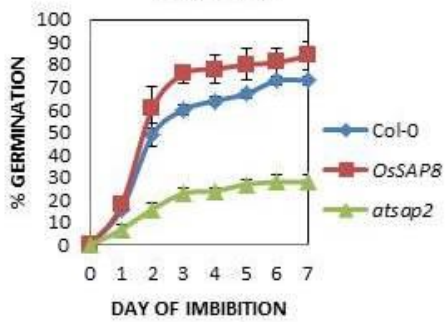

$40 \%$ PEG

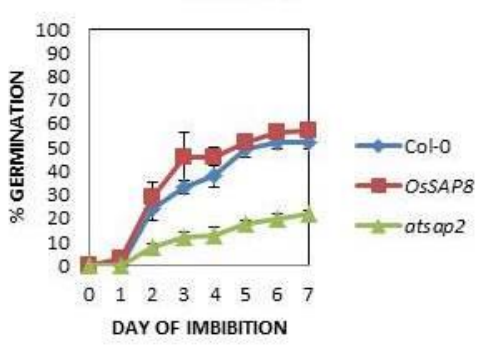

Fig 5. Germination analysis was carried out for Col-0, OsSAP8 and atsap 2 in response to different concentrations of PEG. The graph shows the effect of different concentrations of PEG $(10 \%, 20 \%, 40 \%, 50 \%)$ on the germination of Col-0, OsSAP8 and atsap2 seeds. Water agar was used as a control experiment to observed seed viability (the same seed batch as used in the $\mathrm{NaCl}$ experiment). atsap 2 showed a sensitive germination phenotype to all concentrations of PEG $(10 \%, 20 \%, 40 \%, 50 \%)$ compared to Col-0 and OsSAP8. OsSAP8 showed a tolerant phenotype to $10 \%, 20 \%, 40 \%$ concentrations of PEG, though this was not significantly different to Col-0. 

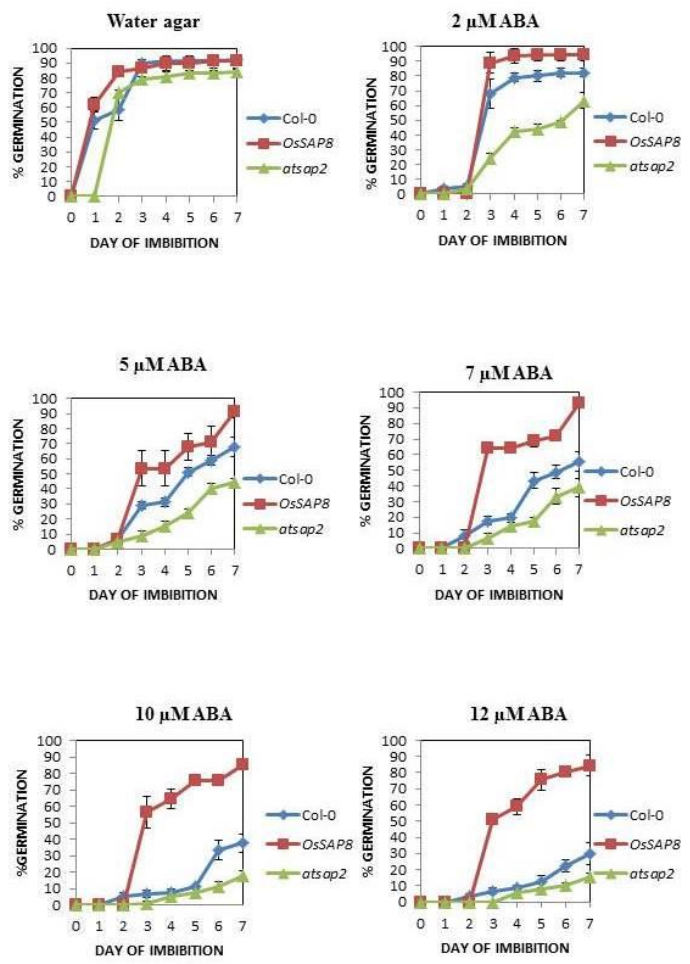

Fig 6. Germination analysis was carried out for Col-0, OsSAP8 and atsap 2 in response to different concentrations of ABA. The graph shows the effect of different concentrations of ABA $(2 \mu \mathrm{M}, 5 \mu \mathrm{M}, 7 \mu \mathrm{M}, 10 \mu \mathrm{M}, 12 \mu \mathrm{M})$ on the germination of Col- 0 , OsSAP8 and atsap2 seeds. OsSAP8 showed an insensitive phenotype to ABA up to $12 \mu \mathrm{M}$ concentration, whilst the atsap2 showed a sensitive phenotype to ABA as low as $2 \mu \mathrm{M}$ ABA. Water agar was used as a control experiment to observe seed viability. OsSAP8 showed an insensitive phenotype to high concentrations of $\mathrm{ABA}$ up to $12 \mu \mathrm{M}$ compared to Col-0 and atsap2.

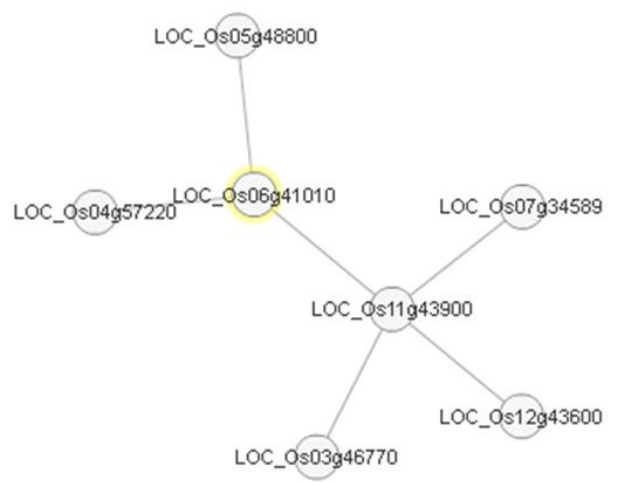

Fig 7. Rice Abiotic Stress Co-expression Network. The co-expression network was carried out using the Rice Oligonucleotide Array Database with the correlation coefficient cutoff at 0.8 and the depth to search co-expressed genes set at 2. The co-expression network result shows 7 nodes with 6 internodes associated with OsSAP8. LOC_Os06g41010 is the OsSAP8 Rice gene Identification (ID).

was built using the Rice Oligonucleotide Array Database available online (Cao et al., 2012). There were six genes that were found to be differentially co-expressed with OsSAP8 during abiotic stress (Fig. 7, Table 1). These genes were divided into two distinct clusters, with one cluster directly co-expressed with OsSAP8 and another co-expressed through one node represented by gene encoded translationallycontrolled tumor protein, putative (LOC_Os11g43900). Among these, one of the genes was drought induced 19 proteins, putative (LOC_Os05g48800). Ontological analysis showed that drought induced 19 proteins; putative plays a role in response to stress and abiotic stimuli. Another gene that was directly co-expressed with OSSAP8 during stress was the ubiquitin-conjugating enzyme, putative
(LOC_osg0457220). This gene is known as E2 enzyme, and plays a role as a catalyst in the covalent attachment of ubiquitin to target proteins. Another gene that was connected to OSSAP8 encodes translationally-controlled tumor protein (TCTP), which is co-expressed together with three other genes: translation initiation factor SUI1, putative, expressed (LOC_Os07g34589) and RNA recognition motif containing protein, expressed encoded by two different gene IDs (LOC_Os12g43600 and LOC_Os03g46770).

\section{Discussion}

In this paper, we describe the role of OSSAP8 in response to multiple abiotic stresses ( $\mathrm{NaCl}$ and PEG) including ABA. We 
show that the overexpression of OSSAP8 enhanced tolerance to salinity stress stimulus $(\mathrm{NaCl})$ compared to its knockout version atsap2. OsSAP8 also showed rapid germination rate compared to Col- 0 and atsap 2 on media supplemented with different concentrations of $\mathrm{NaCl}$. However, as the imbibition day prolonged to day seven, germination percentage for both OSSAP8 and Col-0 did not significantly differ. This may be due to the plant recovery process and stress condition adaptation. Plant abiotic stress mechanisms consist of very complex processes, which include the plant structure itself, post-transcriptional and post-translational modifications, epigenetic regulation, metabolic processes and functional proteins such as molecular chaperons and heat-shock proteins (Hirayama and Shinozaki, 2010; Ruibal et al., 2013). Exposing the plant to a very long stress condition may somehow activate different stress regulatory mechanisms to cope with the situation. In contrast to $\mathrm{NaCl}$, analysis on germination potential of OSSAP8 in response to PEG showed a similar germination curve to Col-0. This may indicate that the OSSAP8 function is more specific during salinity stress compared to osmotic stress. This result also supports our previous salt-stress subtractive hybridization (SSH) library (Hedayati et al., 2015), which shows that this gene is differentially up-regulated in response to salinity stress stimuli. However, this observation is different in atsap2, where an increase in the concentration of $\mathrm{NaCl}$ and PEG resulted in reduced germination. In the absence of atsap 2 function, the seed was totally impaired in response to stress, showing that the SAP family gene is important in the regulation of abiotic stresses. This might be due to the presence of two zinc finger domains, A20 and AN1. In-silico analysis showed that both OSSAP8 in rice and atsap2 in Arabidopsis consist of two zinc finger domains A20 and AN1, respectively. Both domains have been shown to be important during abiotic stresses in several plant species (Paul and Kumar, 2015; Zhang et al., 2015; Giri et al., 2011). Interestingly, these domains also have been shown to be involved in the regulation of plant response to abiotic stresses by modulating the Abscisic acid (ABA) and Giberellin (GA) contents and their signal transduction pathway (Zhang et al., 2015). In the case of abiotic stress in plants, endogenous ABA level will increase to provide a tolerance mechanism against stress. For example, elevated ABA during stress is purported to control stomatal aperture and prevent excessive water loss. This mechanism ensures that plants have adequate water for survival, especially during drought. Ethylene is also another plant hormone that regulates plant response to abiotic stress, besides having a role in the regulation of seed germination in conjunction with ABA (Jun Tao et al., 2015; Linkies et al., 2009).

The co-expression network also showed that several genes connected to OSSAP8 are important for allowing plants to cope with abiotic stresses. One such gene is the ubiquitinconjugating enzyme, putative (LOC_osg0457220). Previous studies have shown that the ubiquitin pathway also has a role in the regulation of plant response to stress (Stone, 2014). The proteolysis event is part of the mechanism that regulates the expression of certain genes or protein activity which are involved in plant stress response. Other than that, the gene encodes for translationally-controlled tumor protein (TCTP), which is also found in the network. This gene has been identified to be involved in calcium binding and microtubule stabilization. Previous studies have shown that calcium ions $(\mathrm{Ca} 2+)$ have an important role during abiotic stress. The $\mathrm{Ca} 2+$ has been shown to be involved in the abiotic stress signaling cascade as a secondary messenger. The presence of calcium enhanced plant response to stress stimuli therefore triggering the activation of transduction pathways and downstream target genes (Das and Pandey, 2010; Tuteja and Sopory, 2008).

An interesting finding involved germination media supplemented with different concentrations of ABA. The overexpression of OsSAP8 showed an insensitive germination phenotype to high concentrations of $\mathrm{ABA}$, while the atsap2 loss-of-function mutant showed a sensitive phenotype to ABA. This sensitive phenotype can be observed on media supplemented with ABA concentration as low as 2 $\mu \mathrm{M}$. A previous study has shown that ABA plays an important role to prevent seed germination and maintain seeds in a dormant state, while GA acts to antagonize ABA in the regulation of seed dormancy to germination (Bewley, 1997; Kucera et al., 2005). The ABA signaling pathway components have been identified via several experiments. One of the components is a negative regulator of $\mathrm{ABA}$ signaling which is PROTEIN PHOSPHATASE 2C (PP2C). This component acts by inhibiting the activity of protein kinase (SnRK2) (Nambara et al., 2010). PP2C is a negative regulator of $\mathrm{ABA}$ signaling. In the absence of $\mathrm{ABA}$, this component acts by inhibiting the downstream target, therefore inactivating the $\mathrm{ABA}$ response. However, in the presence of ABA, the PP2C becomes inactive and releases the SnRK2 to phosphorylate the downstream target for ABA activation genes to be functional. Loss-of-function mutation of PP2C showed a sensitive phenotype to high concentration of ABA. Another report has also shown that overexpression of PP2C like protein in rice, OSPP108 had an insensitive phenotype to ABA (Singh et al., 2015). This insensitive phenotype in the overexpression line is similar to that of our overexpression OSSAP8 phenotypically. However, the sequence comparison between OSSAP8 and PP2C like protein in rice, OSPP108 does not share any significant similarity in the protein sequences. However, the result of the ABA sensitivity test shows that the OsSAP8 and its orthologues atsap2 may have a role in ABA signaling during germination and also in response to stress. More experiments are required to confirm the relationship between OSSAP8 and ABA.

\section{Materials and methods}

\section{Bioinformatics analysis}

The co-expression network analysis of OsSAP8 was performed using the Rice Oligonucleotide Array Database. Gene ontology enrichment analysis was carried out via the Gene Ontology Consortium. Protein sequence alignment was performed using Clustal Omega software, available at EBIEMBL.

\section{Plant material and stress treatment}

Oryza sativa MR219 were obtained from the Malaysian Agricultural Research and Development Institute (MARDI). The rice seeds were immersed in water for two days before being sown on soil and grown in a glasshouse for two weeks to reach the seedling stage. The rice seedlings were then treated with $150 \mathrm{mM} \mathrm{NaCl}$ to mimic salinity stress in soil. This amount of $\mathrm{NaCl}$ is enough to induce stress in MR219 and to enhance the expression of OsSAP8 in MR219 rice samples for gene isolation (Hedayati et al., 2015). Three replicate samples were collected and immediately frozen in liquid nitrogen for RNA extraction preparation. The gene ID for the OSSAP8 gene in the rice genome database is LOC_Os06g41010. 
Another plant used in this experiment was the T-DNA mutant Arabidopsis line. The gene mutation in Arabidopsis is an orthologue to OsSAP8, which is atsap2. atsap2 T-DNA knockout seeds (SALK_008677C) from homozygous lines were ordered from Nottingham Arabidopsis Stock Centre (NASC). The AGI code for the AtSAP2 gene in Arabidopsis is AT1G51200.

\section{RNA extraction, DNase digestion, and cDNA synthesis}

RNA was extracted using TRIzol reagent as described by the manufacturer (Life Technologies). Ethanol precipitation was carried out to further purify the RNA. DNase digestion was carried out using a TURBO DNase Kit (Ambion). RNA was converted to cDNA using a First Strand cDNA Synthesis Kit (Maxima). cDNA were then stored at $-80^{\circ} \mathrm{C}$.

\section{Amplification of OsSAP8 and cloning}

PCR was performed with Q5 High Fidelity DNA Polymerase using the cDNA template. The following primer was used for PCR:

OsSAP8_F:5'-GGGGACAAGTTTGTACAAAAAAGCAGG CTTCATGGAGCACAAGGAGACT-3' and OsSAP8_R: 5'-GGGGACCACTTTGTACAAGAAAGCTGGGT CCTAAATTTTGTCAAGTTTCTCC-3'.

The full sequence of OsSAP8 was cloned into Gateway vectors using $\mathrm{BP}$ and LR Clonase (Gateway Cloning Technology), as described by the manufacturer. Using BP clonase, donor vector, pDONR221 was used to generate entry clone harbouring OSSAP8. Entry clone was transformed into competent E.coli Top10 using the heat-shock method. Selection was carried out using $50 \mu \mathrm{g} / \mathrm{ml} \mathrm{Kanamycin} \mathrm{and}$ colony PCR. The next cloning step was carried out using the LR clonase, plasmid DNA from positively transformed $E$. Coli were cloned into the destination vector, pB2GW7 under 35S CaMV promoter (35S::OsSAP8) (Liang et al., 2013). The expression clone was transformed into E.coli Top 10 using the heat-shock method. Selection was carried out using $75 \mu \mathrm{g} / \mathrm{ml}$ Spectinomycin. Expression clones harbouring $O S S A P 8$ were confirmed using PCR and sent for sequencing. Expression clones harbouring OsSAP 8 were then transformed into Agrobacterium tumefaciens strains GV3101 prior to Arabidopsis transformation. Underlined in the primer sequences were gateway adaptor sequences for the gateway cloning system.

\section{Agrobacterium-mediated transformation in Arabidopsis}

Agrobacterium tumefaciens harbouring the 35S::OsSAP8 construct were transformed into Columbia- 0 via the floral-dip method (Zhang et al. 2006). Bacterial culture was supplemented with $5 \%$ sucrose and $0.05 \%$ silwet- 77 prior to inflorescence dipping.

\section{Screening of OsSAP8 positive transformant}

$\mathrm{T}_{1}$ seeds were screened for positive transformants with 150 $\mathrm{mg} / \mathrm{ml}$ BASTA. Genomic DNA was extracted from positive transformants $\left(\mathrm{T}_{1}\right)$ and a PCR reaction was performed to validate the presence of $O S S A P 8$. The following primer was used for the PCR: 35S F: 5'- TCCCACTATCCT TCGCAAGACC -3 ' and $O S S \bar{A} P 8$ R: 5'-CTAAATTTTGTC AAGTTTCTCC-3'. Seeds from $T_{1}$ plants were then collected and sown on soil until $\mathrm{T}_{3}$ seeds were obtained. $\mathrm{T}_{3}$ seeds were used for functional studies.

\section{Semi-quantitative RT-PCR of atsap2 mutants}

Validations of atsap2 expression were confirmed by semiquantitative RT-PCR. Total RNA was extracted using the QIAGEN RNeasy Plant Mini Kit and converted into cDNA using the First Strand cDNA Synthesis Kit Maxima. Reverse Transcriptase PCR (RT-PCR) was done by using these primers; atsap2_F: 5'-CTGCGGTTTCTTTGGAAGCG-3', atsap2_R: 5'-CTTGGTCCCTTTGGTTTCGC-3'.

\section{Germination test}

Seeds of OsSAP8, atsap2 and wild-type were plated onto water agar media (for control) and for each of the treatments $(\mathrm{NaCl}, \mathrm{PEG}, \mathrm{ABA})$. These $1 / 2 \mathrm{MS}$ media contained $0.4 \mathrm{~g}$ MES, $2.2 \mathrm{~g}$ Murashige and Skoog, $5 \mathrm{~g}$ sucrose and $3 \mathrm{~g}$ gelrite and were adjusted in a range of $\mathrm{pH} 5.7$ to 5.8. The seeds were incubated in the dark at $4^{0} \mathrm{C}$ for 4 days. After incubation, seeds were kept at $25^{\circ} \mathrm{C}$ and germination rates were assessed for 7 days. The rupture of the micropylar endosperm is considered as germination of Arabidopsis thaliana seeds.

\section{Conclusion}

Here, we have shown that regulating the expression of rice STRESS ASSOCIATED PROTEIN 8 (OSSAP8) can alter tolerance to multiple abiotic stresses. Impairment in the gene's expression led to an increased susceptibility of the plant to multiple abiotic stresses including ABA. This might be related to the two zinc finger domains embedded in the OsSAP8 sequence and its orthologue, atsap2. These two domains have been characterized and shown to regulate plant abiotic stress response. We also showed that enhanced expression of OSSAP8 gene increased seed insensitivity towards $\mathrm{ABA}$ and vice versa. It is interesting to look at the relationship between $O S S A P 8$ and ABA in the regulation of environmental stress, as $\mathrm{ABA}$ is a major plant hormone involved in abiotic stress response.

\section{Acknowledgements}

Financial assistance was provided by Universiti Kebangsaan Malaysia via Geran Galakan Penyelidik Muda (GGPM-2013034). We thank Prof. Wan Kiew Lian for comments on the manuscript.

\section{References}

Atkinson NJ, Jain R, Urwin PE (2015) The response of plants to simultaneous biotic and abiotic stress. Combined Stresses in Plants. 181-201.

Bewley JD (1997) Seed germination and dormancy. Plant Cell. 9:1055-1066.

Cao P, Jung KH, Choi D, Hwang D, Zhu J, Ronald PC (2012) The rice oligonucleotide array database: an atlas of rice gene expression. Rice. 5:17.

Cattivelli L, Rizza F, Badeck F-W, Mazzucotelli E, Mastrangelo AM, Francia E, Mare C, Tondelli A, Stanca AM (2008) Drought tolerance improvement in crop plants: an integrated view from breeding to genomics. Field Crop Res. 105(1): 1-14.

Das R, Pandey GK (2010) Expressional analysis and role of calcium regulated kinases in abiotic stress signaling. Curr Genomics. 11(1): 2-13.

Deinlein U, Stephan AB, Horie T, Luo W, Xu G, Schroeder JI (2014) Plant salt-tolerance mechanisms. Trends Plant Sci. 19(6): 371-379. 
Giri J, Vij S, Dansana PK, Tyagi AK (2011) Rice A20/An1 zinc-finger containing stress- associated proteins (Sap1/11) and a receptor-like cytoplasmic kinase (Osrlck253) interact via A20 zinc-finger and confer abiotic stress tolerance in transgenic arabidopsis plants. New Phytol. 191(3): 721-732.

Godfray HCJ, Beddington JR, Crute IR, Haddad L, Lawrence D, Muir JF, Pretty J, Robinson S, Thomas SM, Toulmin C (2010) Food security: the challenge of feeding 9 billion people. Science. 327(5967): 812-818.

Hedayati P, Hossein HM, Md Isa N, Hwang DJ, Mohd Zain CRC, Imtiaz Uddin M, Zuraida AR, Ismail I, Zainal Z (2015) Construction and analysis of an Oryza sativa (cv. MR219) salinity-related cDNA library. Acta Physiol Plant. 37:91.

Hirayama T, Shinozaki K (2010) Research on plant abiotic stress responses in the post-genome era: past, present and future. Plant J. 61: 1041-1052.

Jun Tao J, Wei Chen H, Biao Ma, Ke Zhang W, Yi Chen S, Song Zhang J (2015) The role of ethylene in plants under salinity stress. Front Plant Sci. 6: 1059.

Kanneganti V, Gupta AK (2008) Over-expression of OsiSAP8, a member of stress associated protein (Sap) gene family of rice confers tolerance to salt, drought and cold stress in transgenic tobacco and rice. Plant Mol Biol. 66(5): 445-462.

Kobayashi Y, Yamamoto S, Minami H, Kagaya Y, Hattori T (2004) Differential activation of the rice sucrose nonfermenting1-related protein kinase2 family by hyperosmotic stress and abscisic acid. Plant Cell. 16:11631177.

Kucera B, Cohn MA, Leubner-Metzger G (2005) Plant hormone interactions during seed dormancy release and germination. Seed Sci Res. 15: 281-307.

Liang X, Peng L, Baek C-H, Katzen F (2013) Single step $\mathrm{Bp} / \mathrm{Lr}$ combined gateway reactions.

Biotechniques. 55(5): 265-268.

Leung J, Merlot S, Giraudat J (1997) The arabidopsis ABSCISIC ACID-INSENSITIVE2 (ABI2) and ABI1 genes encode homologous protein phosphatases $2 \mathrm{C}$ involved in abscisic acid signal transduction. Plant Cell. 9(5):759-71.

Leung J, Bouvier-Durand M, Morris PC, Guerrier D, Chefdor F, Giraudat J (1994) Arabidopsis ABA response gene ABI1: features of a calcium-modulated protein phosphatase. Science. 264(5164):1448-52.

Linkies A, Müller K, Morris K, Turečková V, Wenk M, Cadman CS, Corbineau F, Strnad M, Lynn JR, FinchSavage WE (2009) Ethylene interacts with abscisic acid to regulate endosperm rupture during germination: a comparative approach using Lepidium Sativum and Arabidopsis thaliana. Plant Cell. 21(12): 3803-3822.

Ma Y, Szostkiewicz I, Korte A, Moes D, Yang Y, Christmann A, Grill E (2009) Regulators of PP2C phosphatase activity function as abscisic acid sensors. Science. 324: 1064-1068.

Martin RC, Glover-Cutter K, Baldwin JC, Dombrowski JE (2012) Identification and characterization of a salt stress- inducible zinc finger protein from Festuca arundinacea. BMC Res Notes. 5: 66.

Meyer K, Leube MP, Grill E (1994) A protein phosphatase $2 \mathrm{C}$ involved in ABA signal transduction in Arabidopsis thaliana. Science. 264(5164):1452-5.

Munns R, Tester M (2008) Mechanisms of salinity tolerance. Annu Rev Plant Biol. 59: 651-681.

Munns R (2002) Comparative physiology of salt and water stress. Plant Cell Environ. 25(2): 239-250.

Nambara E, Okamoto M, Tatematsu K, Yano R, Seo M, Kamiya Y (2010) Abscisic acid and the control of seed dormany and germination. Seed Sci Res. 20: 55-67.

Nambara E, Marion-Poll A (2005) Abscisic acid biosynthesis and catabolism. Annu Rev Plant Biol. 56: 165-185.

Park SY, Fung P, Nishimura N, Jensen DR, Fujii H, Zhao Y, Lumba S, Santiago J, Rodrigues A, Chow TF, Alfred SE, Bonetta D, Finkelstein R, Provart NJ, Desveaux D, Rodriguez PL, McCourt P, Zhu JK, Schroeder JI, Volkman BF, Cutler SR (2009) Abscisic acid inhibits type 2C protein phosphatases via the PYR/PYL family of START proteins. Science. 324(5930): 1068-71.

Paul A, Kumar S (2015) An A20/AN1-zinc-finger domain containing protein gene in tea is differentially expressed during winter dormancy and in response to abiotic stress and plant growth regulators. Plant Gene. 1: 1-7.

Ruibal C, Castro A, Carballo V, Szabados L, Vidal S (2013) Recovery from heat, salt and osmotic stress in Physcomitrella Patens requires a functional small heat shock protein PpHsp16.4. BMC Plant Biol. 13:174.

Singh A, Jha SK, Bagri J, Pandey GK (2015) ABA inducible rice protein phosphatase $2 \mathrm{C}$ confers $\mathrm{ABA}$ insensitivity and abiotic stress tolerance in arabidopsis. PLoS ONE. 10(4).

Stone SL (2014) The role of ubiquitin and the 26S proteasome in plant abiotic stress signaling. Front. Plant Sci. 5: 1-10

Tester M, Davenport R (2003) Na+ tolerance and Na+ transport in higher plants. Ann Bot-London. 91(5): 503527.

Tuteja N, Sopory KS (2008) Chemical signaling under abiotic stress environment in plants. Plant Signal Behav. 3(8): 525-536.

Umezawa T, Nakashima K, Miyakawa T, Kuromori T, Tanokura M, Shinozaki K \& Yamaguchi-Shinozaki K (2010) Molecular basis of the core regulatory network in $\mathrm{ABA}$ responses: sensing, signaling and transport. Plant Cell Physiol. 51(11): 1821-1839.

Vij S, Tyagi AK (2007) Emerging trends in the functional genomics of the abiotic stress response in crop plants. Plant Biotechnol J. 5(3): 361-380.

Zhang Y, Lan H, Shao Q, Wang R, Chen H, Tang H, Zhang H \& Huang J (2015) An A20/AN1-type zinc finger protein modulates gibberellins and abscisic acid contents and increases sensitivity to abiotic stress in Rice (Oryza sativa L.). J Exp Bot. 67(1):315-326.

Zhang X, Henriques R, Lin S-S, Niu Q-W, Chua N-H (2006) Agrobacterium mediated transformation of Arabidopsis Thaliana using the floral dip method. Nature Protoc. 1(2): 641-646. 